\title{
A STUDY ON MODAL CHARACTERISTICS OF FLOW SKIRT USING EFFECTIVE YOUNG'S MODULUS
}

\author{
MYUNG JO JHUNG ${ }^{*}$ and YONG BEUM KIM \\ Korea Institute of Nuclear Safety \\ 62 Gwahak-ro, Yuseong-gu, Daejeon, 305-338 Korea \\ *Corresponding author. E-mail : mjj@kins.re.kr \\ Received March 15, 2011 \\ Accepted for Publication June 20, 2011
}

Many innovative design features are employed in the reactor vessel internals of SMART, a small integral-type pressurized water reactor, one of which is the flow skirt, which uniformly distributes flow and horizontally restrains the lower part of the core support barrel. This new design requires a comprehensive investigation of vibration characteristics. Therefore, in this study, modal characteristics of flow skirts are investigated with finite element analysis. Specifically, we investigate how the presence of holes, the presence of three rings attached to the flow skirt, and the thickness of the lowest shell effect vibration characteristics. In addition, the fluid effect is addressed, since the flow skirt is submerged in the fluid.

KEYWORDS : Small and Medium Sized Reactor, Flow Skirt, Effective Young's Modulus, Natural Frequency, Mode Shape

\section{INTRODUCTION}

Various types of advanced small and medium reactors (SMR) are currently under development worldwide, including some which are ready for construction. One advantage of SMRs is that they allow for easy implementation of advanced design concepts and technology. Safety can be significantly enhanced by adopting inherent safety characteristics and passive safety features. Furthermore, the economics of SMRs can be improved by system simplification, modularization, and reduction in the construction time [1].

Korea Atomic Energy Research Institute is developing an advanced SMR called SMART (System-integrated Modular Advanced ReacTor), a small integral-type pressurized water reactor with thermal power of $330 \mathrm{MW}$. SMART represents a coalescence of innovative safety features, indigenous technologies, and other proven technologies for light water reactors [2]. SMART offers substantially enhanced safety thanks to the integral layout of its major components - such as the reactor core, steam generator, coolant pump, and pressurizer-which are integrated within a single pressure vessel. SMART can simultaneously desalinate seawater and generate electricity, which means that SMART can supply both energy and water to large industrial areas or isolated areas such as islands.

SMART's reactor assembly contains major primary systems such as fuel and core, eight steam generators, a pressurizer, four main coolant pumps, and 25 control rod drive mechanisms, all within a single pressurized reactor vessel. The integrated arrangement of these components foregoes the need for large pipe connections between major components of the reactor coolant system, which fundamentally eliminates the possibility of an accident due to a large break and subsequent loss of coolant.

Many innovative design features are also employed in the reactor vessel internals, one of which is the flow skirt for uniformly distributing flow, horizontally restraining the lower part of the core support barrel (CSB), and supporting the core if the fuel assembly drops into the lower head of the reactor vessel due to CSB failure. This kind of new design necessitates a comprehensive investigation of vibration characteristics, which the designer is performing both analytically and experimentally in order to prepare a document for licensing purposes.

Therefore, in this study, the modal characteristics of flow skirts are investigated through the use of finite element analysis. Specifically, we investigate how the vibration characteristics are affected by the presence of holes, the presence of three rings attached to the flow skirt, and the thickness of the lowest shell. In addition, the fluid effect is also addressed, because the flow skirt is submerged in fluid.

\section{ANALYSIS}

\subsection{Finite Element Model}

The physical properties of the shell material are as 
follows: Young's modulus $=191 \mathrm{GPa}$, Poisson's ratio $=0.3$, and mass density $=8110 \mathrm{~kg} / \mathrm{m}^{3}$. Water, with a density of $1000 \mathrm{~kg} / \mathrm{m}^{3}$, is used as the contained fluid. The sound speed in water, $1483 \mathrm{~m} / \mathrm{s}$, is equivalent to the bulk modulus of elasticity, 2.2 GPa.

Two different three-dimensional models are constructed for finite element analysis: one flow skirt with holes and no water, and one flow skirt with water and no holes (Figs. 1 and 2, respectively). For simplicity, the upper flanges and supporting plates are not included in the model. The fluid region is divided into a number of three-dimensional contained fluid elements (FLUID80), with eight nodes having three degrees of freedom at each node. The fluid element FLUID80 is particularly well suited for calculating hydrostatic pressures and fluid/ solid interactions. The circular cylindrical shell is modeled as elastic shell elements (SHELL63) with four nodes.

At the bottom, the fluid boundary conditions are zero displacement and rotation. The nodes connected entirely

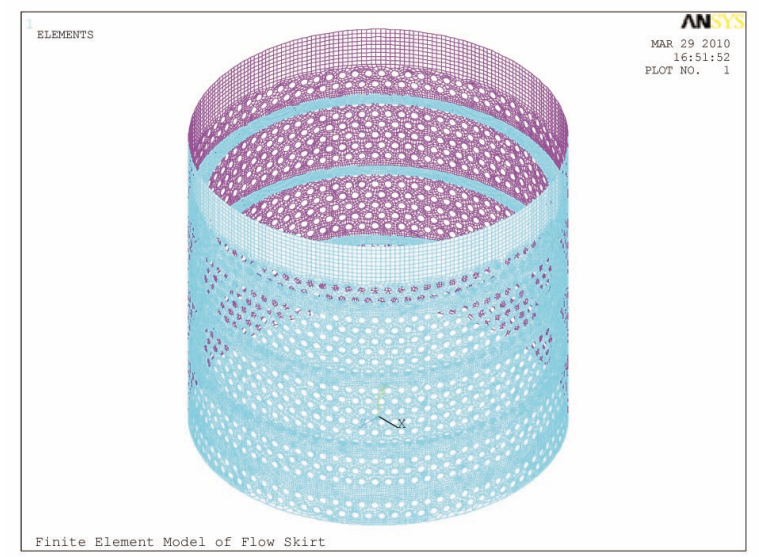

Fig. 1. Finite Element Model of Flow Skirt with Holes and no Water

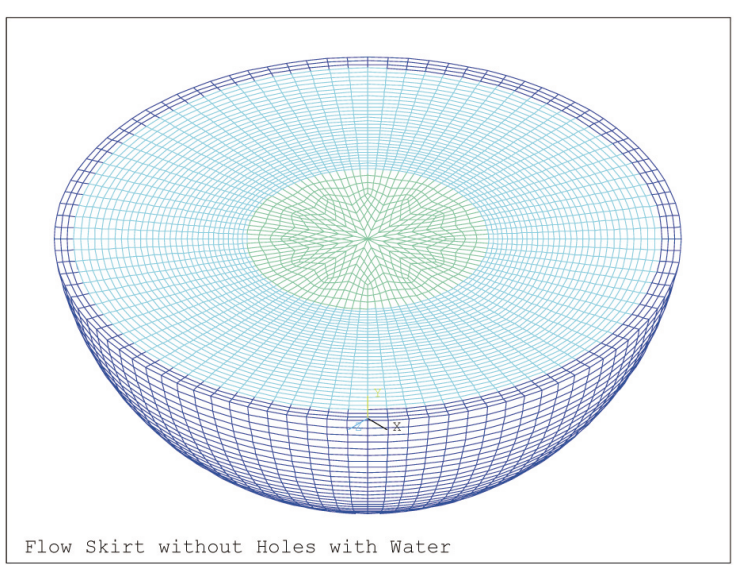

Fig. 2. Finite Element Model of Flow Skirt with Water and no Holes by the fluid elements are free to move arbitrarily in the three-dimensional space, with the exception of those which are restricted to motion in the bottom surface of the fluid. The radial velocities of the fluid nodes along the wetted shell surfaces coincide with the corresponding velocities of the shells. The bottom of the flow skirt is welded to the lower head of the reactor vessel by a 30 degree gap in the circumference, the nodes of which are simulated to move at the same time in all degrees of freedom.

\subsection{Modal Analysis}

Several finite element analyses using a commercial computer code ANSYS 12.0 [3] are performed to get the natural frequencies. The Block Lanczos method is used for the eigenvalue and eigenvector extractions of the finite element model, which meets the requirements for a large symmetric eigenvalue problem [4]. Typically, this solver is applicable to the type of problem solved using the subspace eigenvalue method. However, at a faster convergence rate, the solver is very useful for finding all of the exact symmetric modes necessary to define the dynamic characteristics of the shell. In this case, several sloshing modes of a fluid appear at the same time, so they should be excluded for only the shell modes.

\subsection{Equivalent Material Properties}

The effective elastic constants for the perforated shell are suggested by performing several finite element analyses with respect to the ligament efficiencies [5]. Because the elastic constant is proportional to the square of the natural frequency, we had to find multipliers for Young's modulus of solid shell to match the natural frequencies of perforated shell, with original properties using the relations between natural frequency and Young's modulus. Then, the effective elastic constant for each ligament efficiency is averaged for all modes, thus generating the effective elastic constants for the modal characteristics of a perforated shell with a triangular penetration pattern, as:

$$
\begin{aligned}
\frac{E^{*}}{E}= & 0.1610+1.7421 \eta-2.0365 \eta^{2} \\
& +2.2733 \eta^{3}-1.1471 \eta^{4}
\end{aligned}
$$

where $\mathrm{E}, \mathrm{E}^{*}$ and $\eta$ are original Young's modulus, effective Young's modulus, and the ligament efficiency, respectively. For frequency errors of the perforated shell to be within $10 \%$, the effective elastic constant defined in Equation (1) should be limited only for $0.5 \leq \eta \leq 0.8$, because the constant was obtained by averaging effective elastic constants according to the ligament efficiency [5].

\section{RESULTS AND DISCUSSION}

Natural frequencies of the flow skirt are obtained with respect to various conditions, and they are categorized as 
axial and circumferential modes, where the symbol $\mathrm{m}$ represents the number of axial mode and the symbol $n$ represents the number of circumferential mode. Two different natural frequencies for certain modes are obtained, because the fixed boundary conditions at the bottom of the shell are not fully symmetrical with every 30 degrees fixed in the circumferential direction. The natural frequency variations for a flow skirt with holes and no water, and for one with water and no holes are shown in Figs. 3 and 4, respectively.

Several sensitivity runs are performed for holes, water, rings, and thickness of the lowest shell, and their results are compared. Because a flow skirt consists of a perforated shell with many holes, the hole effect is investigated. Natural frequency comparisons between flow skirts with and without-holes are shown in Fig. 5, and the normalized values of flow skirts with holes to those without holes are shown in Fig. 6. For the lower axial modes, the hole effects are almost negligible, but as the axial mode increases, the effect becomes significant. By including holes in the flow skirt model, the natural frequencies decrease about $12 \%$ for the first three axial modes.

A flow skirt has three inner rings attached to it, and these will stiffen the structure and increase the natural
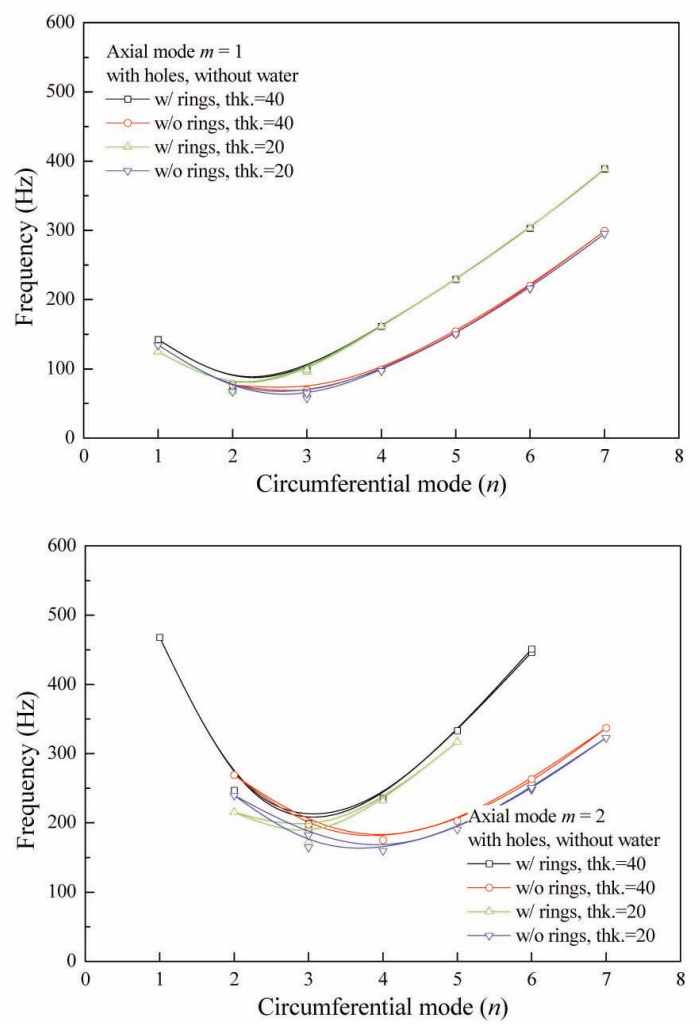

Fig. 3. Natural Frequencies of Flow Skirt with Holes and no Water
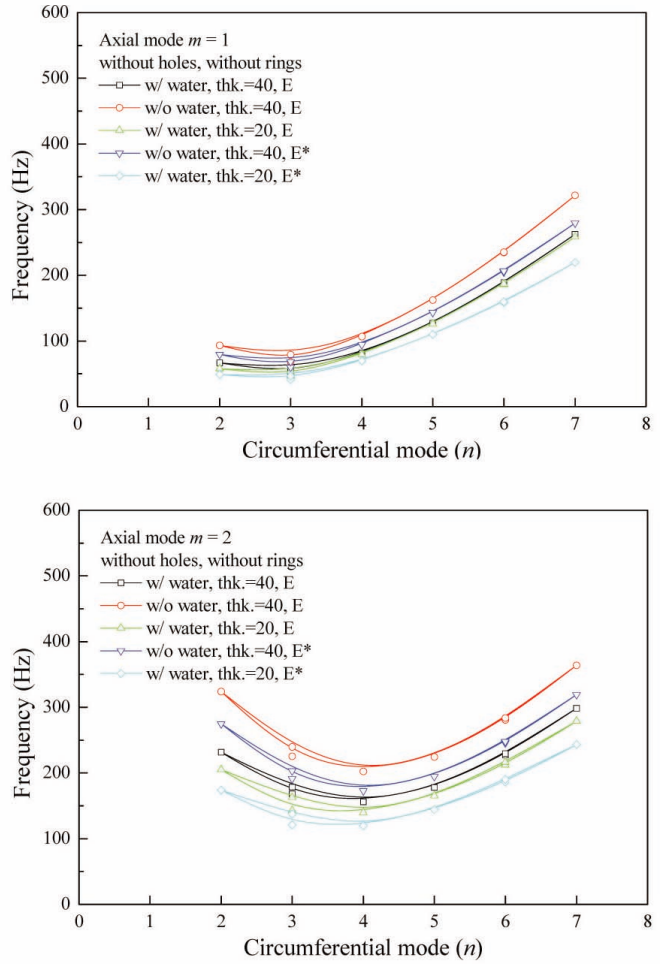

Fig. 4. Natural Frequencies of Flow Skirt without Holes, Both with and without Water

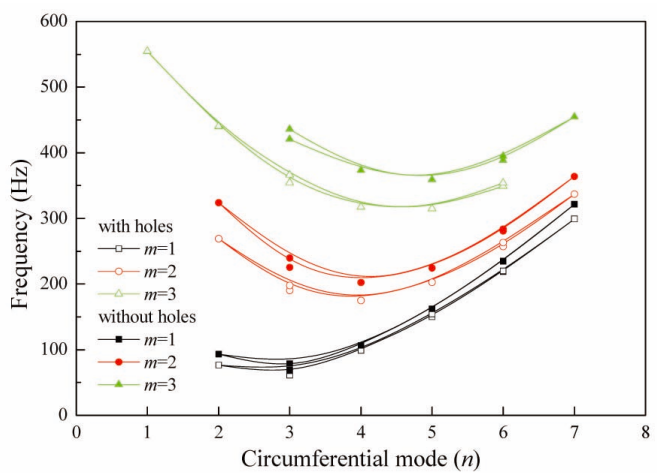

Fig. 5. Effect of Holes on the Natural Frequencies

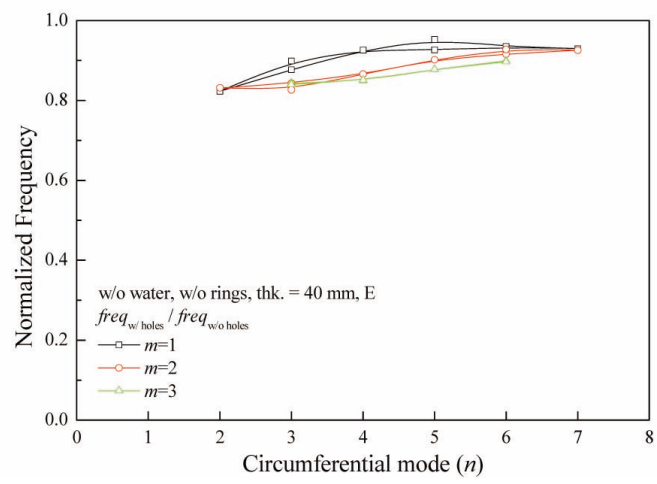

Fig. 6. Normalized Natural Frequencies of Flow Skirt Considering Hole Effect 


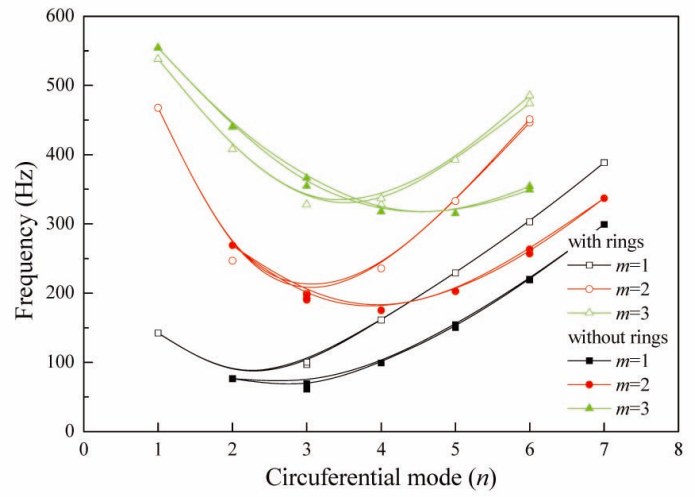

Fig. 7. Effect of Rings on the Natural Frequencies
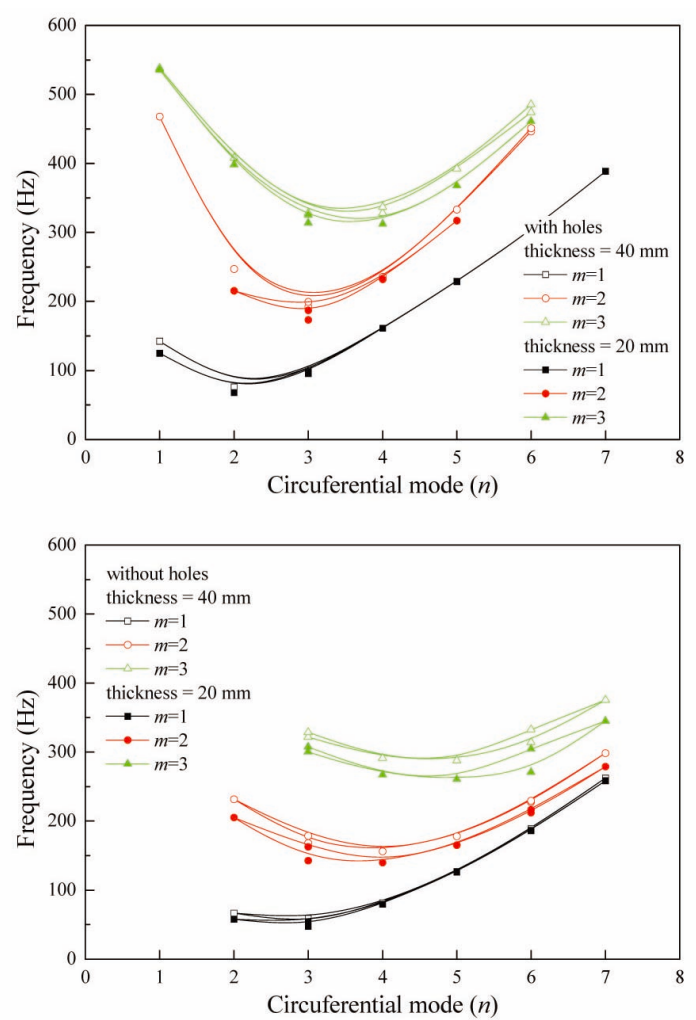

Fig. 8. Effect of Thickness at the Lowest Level of Cylinder on the Natural Frequencies

frequencies, as shown in Fig. 7. For the lower circumferential modes, the natural frequencies are virtually identical, but as the circumferential modes increase, the natural frequencies shows too much increase, which should not be neglected. A good design for increasing the natural frequency is to attach the inner rings to the shell type of structure.

The flow skirt considered in this study has a thickness of $40 \mathrm{~mm}$, except at the lowest level of the shell, where the thickness is $20 \mathrm{~mm}$. This effect is investigated by comparing the natural frequencies, as shown in Fig. 8. For

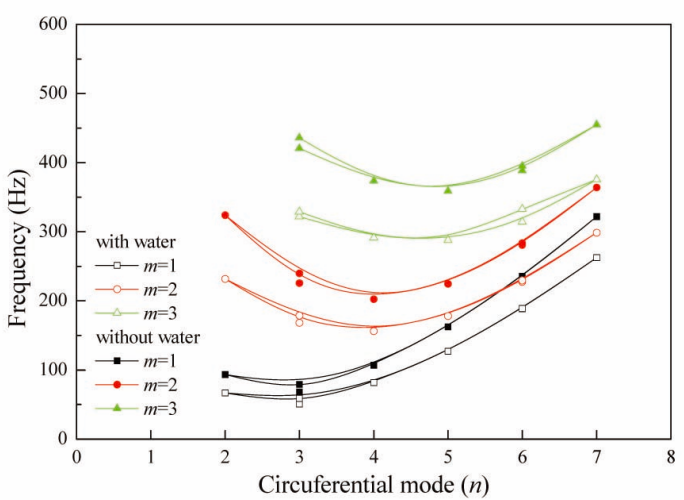

Fig. 9. Effect of Water on the Natural Frequencies

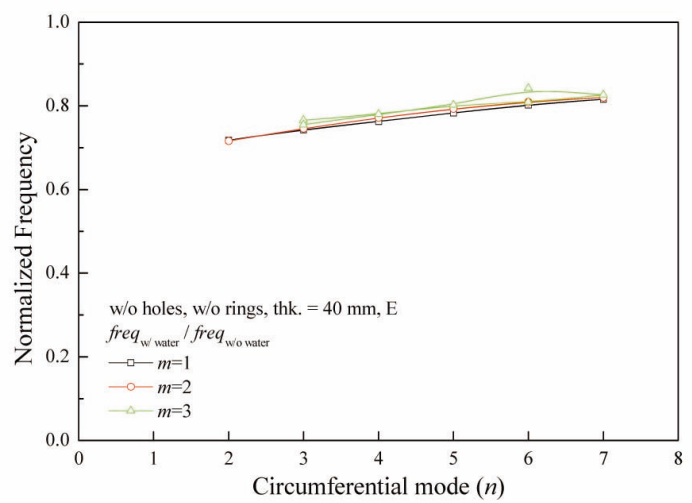

Fig. 10. Normalized Frequencies of Flow Skirt Considering Water Effect without Holes

the first axial modes, the natural frequencies are almost the same, and the second and the third axial modes have differences within about $10 \%$.

If the structure is submerged in fluid, the natural frequencies decrease due to the fluid effect, which can be explained by added mass and/or fluid coupling effect $[6,7]$. The natural frequency comparisons between flow skirts with and without water are shown in Fig. 9. The effect of fluid on the natural frequencies of the flow skirt that is wetted with fluid can be assessed using the normalized frequency, which is defined as the natural frequency of a structure in contact with a fluid divided by the corresponding natural frequency in air. The normalized natural frequencies have values between one and zero due to the added mass effect of fluid. Figure 10 shows the normalized natural frequencies with ranges between 0.71 and 0.84 . As the circumferential wave number increases, the normalized natural frequencies increase because of the gradual reduction of the relative added mass effect. Therefore, an increase of circumferential wave numbers causes an increase in the normalized natural frequencies for all cases.

The flow skirt has a ligament efficiency $\eta$ of about 0.5 , so the effective Young's modulus $E^{*} / E$ can be calculated using Equation (1). If this effective Young's 


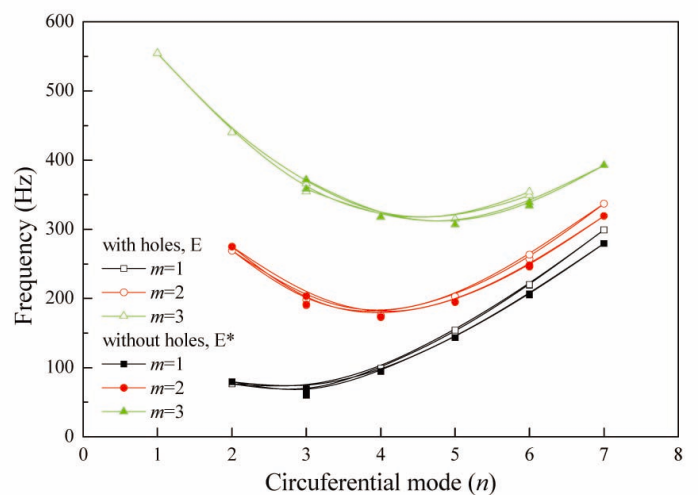

Fig. 11. Comparison of Natural Frequencies between Perforated Shell with Original Young's Modulus and Solid Shell with Effective Young's Modulus

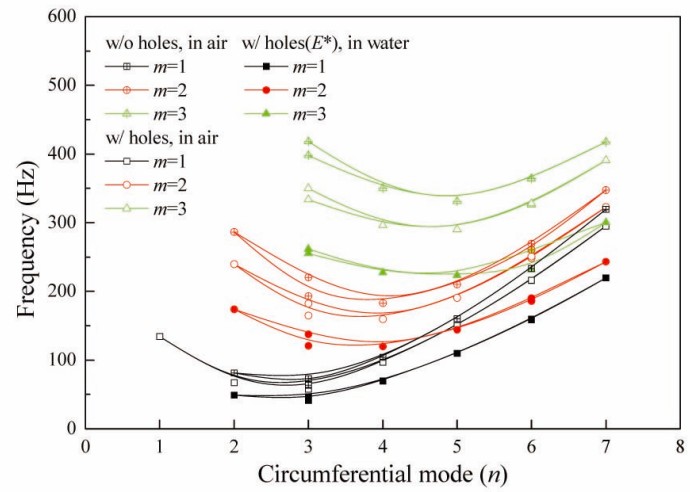

Fig. 12. Natural Frequencies of Flow Skirt without Rings and Thickness $=20 \mathrm{~mm}$

modulus is used for the solid shell, the natural frequencies should be approximately the same as those of the perforated shell with original Young's modulus, as shown in Fig. 11. Therefore the flow skirt in water can be modeled as a solid shell using the effective Young's modulus.

Figure 12 shows the natural frequencies of a flow skirt without rings where the lowest shell thickness is 20 $\mathrm{mm}$. Here, the shell with holes has original property and the shell without holes has effective property, so that the same hole effects are considered. Because the shell without holes can be submerged in fluid, the final natural frequencies are summarized (as in Fig. 12) for the case with water, without holes, and effective Young's modulus. Figure 13 shows the normalized natural frequencies of the flow skirt without rings with the lowest shell thickness of $20 \mathrm{~mm}$, with respect to the hole effect, fluid effect, and both effects with the average normalized values of 0.89 , 0.75 , and 0.67 , respectively.

\section{CONCLUSIONS}

This study investigates the modal characteristics of a

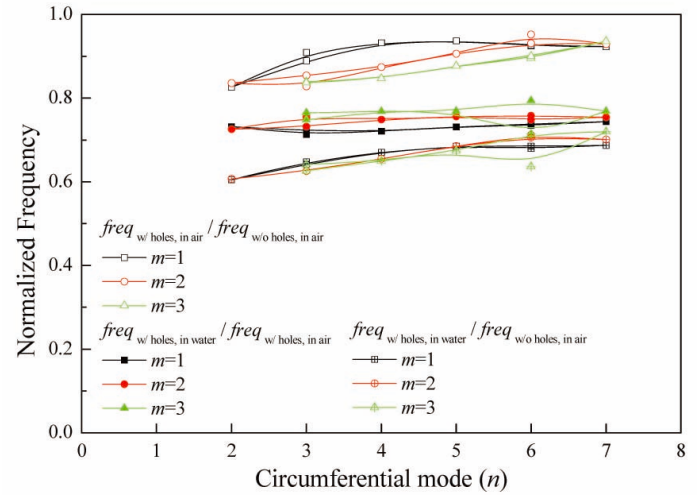

Fig. 13. Normalized Natural Frequencies of Flow Skirt without Rings and Thickness $=20 \mathrm{~mm}$

flow skirt for SMART, which is under development in Korea. Various sensitivity studies are performed to determine how the thickness of the lowest shell, the presence of three rings attached to the flow skirt, and the presence holes affect the vibration characteristics. Also, the fluid effect is investigated using the method of effective material property. We generated the following conclusions:

- The first natural frequency of the flow skirt is $68 \mathrm{~Hz}$ in air and $41 \mathrm{~Hz}$ in water.

- Even when the thickness of the lowest shell is half that of other levels of the shell, the effects on the natural frequencies are almost negligible.

- Having the three rings attached inside the flow skirt increases natural frequencies too much for the higher circumferential modes, which should not be neglected.

- Including the hole effect reduces the natural frequencies to about $89 \%$ of the without-hole natural frequencies.

- Including the fluid effect reduces the natural frequencies to about $75 \%$ of the in-air natural frequencies.

\section{REFERENCES}

[1] IAEA, Innovative Small and Medium Sized Reactors: Design Features, Safety Approaches, and R\&D Trends, IAEA-TECDOC-1451, International Atomic Energy Agency, Vienna (2005).

[2 ] Kim, K.M., Lee, B.I., Cho, H.H., Park, J.S., Chung, Y-J., "Numerical study on thermo-hydrodynamics in the reactor internals of SMART," Nuclear Engineering and Design, Vol.241, Issue 7, pp.2536-2543 (2011).

[3] ANSYS, Inc., Theory Reference for ANSYS and ANSYS Workbench Release 12.0, Canonsburg, PA (2009).

[4 ] Grimes, R.G., Lewis, J.G., Simon, H.D., “A Shifted Block Lanczos Algorithm for Solving Sparse Symmetric Generalized Eigenproblems," SIAM Journal on Matrix Analysis and Applications, Vol.15, No.1, pp.228-272 (1994).

[ 5 ] Jhung, M.J., Yu, S.O., "Study on modal characteristics of perforated shell using effective Young's modulus," Nuclear Engineering and Design, Vol.241, pp.2026-2033 (2011). 
[6] Jhung, M.J., Choi, Y.H., Ryu, Y.H., "Free vibration analysis of circular plate with eccentric hole submerged in fluid," Nuclear Engineering and Technology, Vol.41, No.3, pp.355-364 (2009).
[ 7 ] Jhung, M.J., Kim, W.T., Ryu, Y.H., "Dynamic characteristics of cylindrical shells considering fluid-structure interaction," Nuclear Engineering and Technology, Vol.41, No.10, pp.1333-1345 (2009). 